\title{
A Parallel and Distributed Analysis Pipeline for Performance Tree Evaluation
}

\author{
Darren K. Brien, Nicholas J. Dingle, William J. Knottenbelt, Harini Kulatunga and Tamas Suto \\ Department of Computing, Imperial College London \\ 180 Queen's Gate, SW7 2BZ London, United Kingdom \\ \{dkb03,njd200,wjk, hkulatun, suto\}@doc.ic.ac.uk
}

\begin{abstract}
Performance Trees are a unifying framework for the specification of performance queries involving measures and requirements. This paper describes an evaluation environment for Performance Trees comprising a client-side Performance Query Editor, incorporated as a module of the PIPE2 Petri net tool, and a cluster-based server-side evaluation engine. The latter combines the capabilities of a number of parallel and distributed analysis tools.
\end{abstract}

\section{Introduction}

Performance Trees (PTs) provide the means to express a wide variety of queries on stochastic models [1]. Graphically, PTs are represented as tree structures consisting of operation and value nodes. Operation nodes represent performance-related concepts, e.g. the calculation of a passage time density, while value nodes represent the inputs to these operations. PTs are extensible, either by the addition of new operators, or via a macro mechanism based on the set of existing operators.

The PIPE2 tool [2] provides a graphical interface for the creation, editing and analysis of Generalised Stochastic Petri net (GSPN) models, and also permits further functionality to be added by user-designed modules. We have implemented a Performance Query Editor (PQE) module for the graphical specification of performance queries using PTs. These queries are submitted by the PQE module to a cluster-based query evaluation engine, which harnesses a number of performance analysis tools. These are capable of

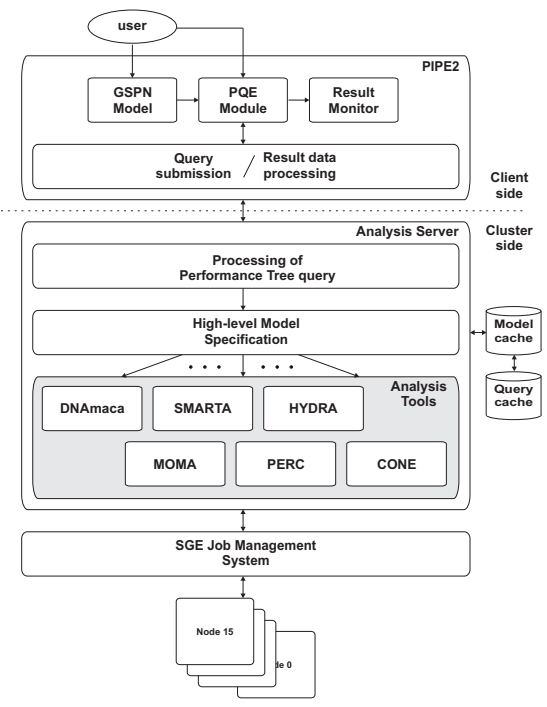

Fig. 1. Performance Tree analysis pipeline

evaluating GSPN models for quantities such as steadystate probabilities, moments and distributions of response times and transient measures. The results from the evaluation of a PT query using these tools are displayed to the user by the PQE module. The result is a comprehensive system modelling, performance query design and performance query evaluation pipeline.

\section{Performance Query Editor Module}

The PQE module enables the construction of a Performance Tree query relating to the current GSPN model as shown in Figure 2. Note that the user sees not only the graphical tree representation, but also a natural language equivalent of the current query. 


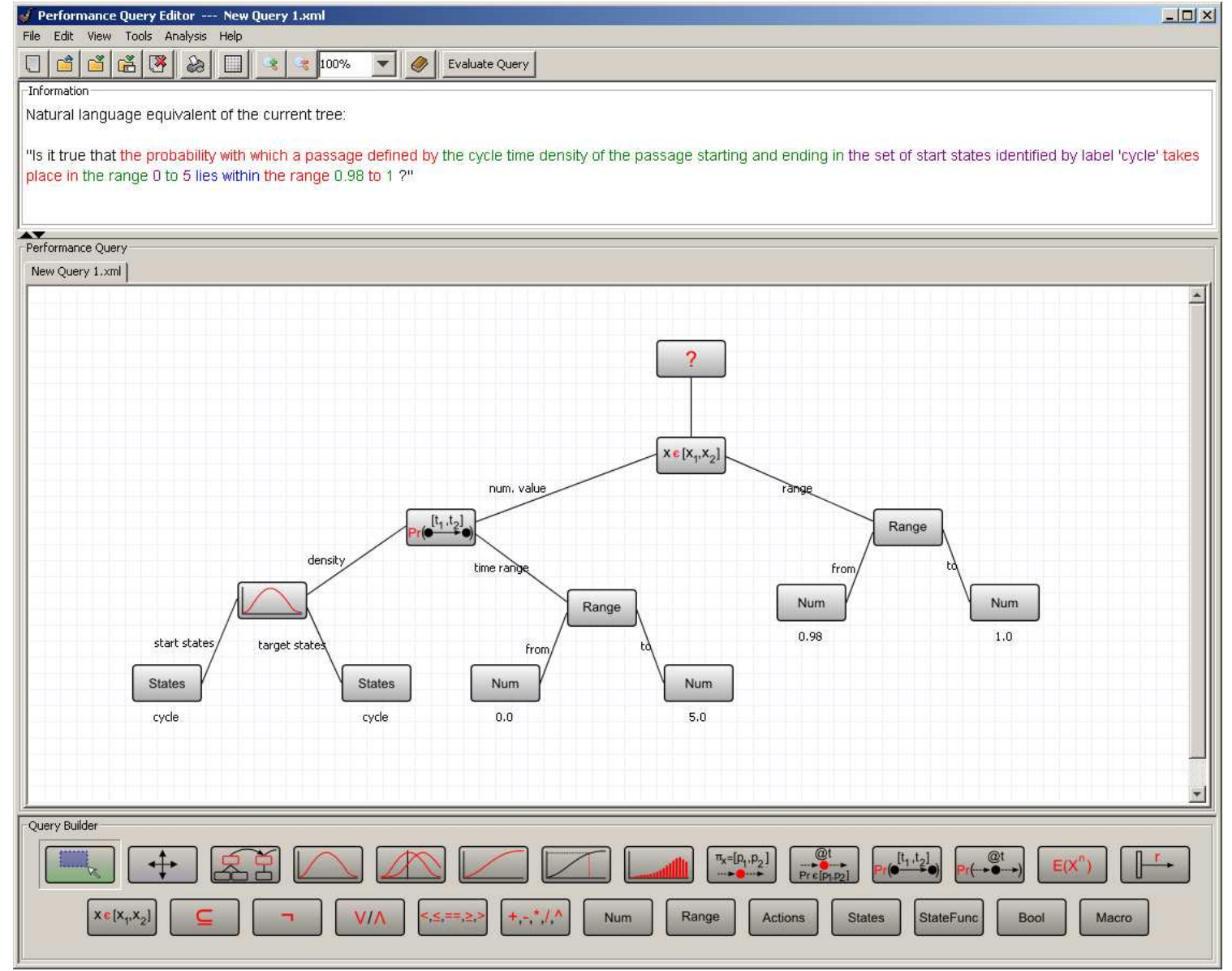

Fig. 2. The Performance Tree query editor module for PIPE2

\section{Analysis Server}

Once a GSPN model and a corresponding Performance Tree query have been defined, they may be submitted for evaluation. When this happens, the PQE module communicates, in serialised object form, a data representation of the query and the model to the Analysis Server.

The Analysis Server then schedules the nodes of the PT for evaluation, taking into account any dependencies that need to be observed, and using a disk-based cache to avoid the evaluation of previously computed queries. Each uncached non-trivial operation node results in a (possibly distributed) task being submitted to the Sun Grid Engine (SGE) for execution on the cluster back-end. Each task invokes an analysis tool, such as DNAmaca, SMARTA, HYDRA, MOMA, PERC and CONE [3]. These tools provide the ability to calculate steady-state distributions, passage time densities and distributions, their convolutions, per- centiles and higher moments, transient measures etc. As results of node evaluations become available, the analysis server returns them to the PQE module, which displays them in the Result Monitor.

\section{References}

[1] T. Suto, J. T. Bradley, and W. J. Knottenbelt, "Performance trees: Expressiveness and quantitative semantics," in Proc. $4^{\text {th }}$ Intl. Conf. on the Quantitative Evaluation of Systems (QEST), Edinburgh, United Kingdom, September 2007, pp. 41-50.

[2] PIPE: Platform-Independent Petri net Editor - http://pipe2. sourceforge.net.

[3] D. K. Brien, N. J. Dingle, W. J. Knottenbelt, H. Kulatunga, and T. Suto, "Performance trees: Implementation and distributed evaluation," in PDMC'08, Proc. $7^{\text {th }}$ Intl. Workshop on Parallel and Distributed Methods in Verification, Budapest, Hungary, March 2008, Work in Progress Report.

A note on tool availability: The PQE module is currently available via CVS checkout from the PIPE2 SourceForge website [2]; a mainstream release is expected shortly. 